\title{
Peripherien und Zentralitäten: Geographien von Covid-19
}

Überlegungen zu Roger Keils Beitrag zu der translokalen Vorlesungsreihe „Geographien von Covid-19“ sowie der s u b \u r b a n-Debatte zum „Ende des Städtischen“
Stefan Höhne, Boris Michel ,Das Ende des Städtischen? Pandemie, Digitalisierung und planetarische Enturbanisierung'

und

Roger Keil ,Covid Urban: Dimensionen der Peripherie in der städtischen Gesellschaft'

\section{Einleitung}

Covid-19 macht soziale und ökonomische Ungleichheiten global sichtbar und verschärft sie.[1] Wie die aktuelle s u b $\backslash \mathrm{u}$ r b a n-Debatte zum „Ende des Städtischen" mit dem Aufschlag von Stefan Höhne und Boris Michel (2021: 146) verdeutlicht, haben wir es mit einer „weltweiten massiven ökonomischen, sozialen und kulturellen Krise der Städte“ zu tun, die sich in gewisser Weise, so die provokante These der beiden, „als ein Prozess der Enturbanisierung beschreiben" lasse. Unbestritten geht mit der CoronaKrise eine Akzentuierung der gesellschaftlichen Spaltungen entlang von class, race und gender einher. Die soziale Schere öffnet sich und politische Polarisierungen, autoritäre sowie antidemokratische Tendenzen treten akzentuierter hervor (Mullis 2020). Die Ungleichheiten sind relational verwoben und haben eine Geographie. Im Gefüge einer kapitalistisch organisierten und planetar urbanisierenden Welt (Brenner/Schmid 2015) gilt, so Samantha Biglieri, Lorenzo De Vidovich und Roger Keil (2020: 1; Übers. d. A.): ,[W]o das Virus konzentriert auftritt, da findet man die Peripherie, in der Stadt und in der Gesellschaft“. Keil (2021a) konkretisiert diese Perspektive in seinem Vortrag vom 26. Januar $2021 \mathrm{im}$ Rahmen der translokalen Vorlesungsreihe „Geographien von Covid-19“ sowie in seinem Kommentar zu Höhne und Michel (Keil 2021b).

Mit Bezügen zur s u b $\backslash \mathrm{u} r \mathrm{~b}$ a n-Debatte werde ich im Folgenden zunächst Peripherisierungen der Pandemie in Deutschland diskutieren. Dafür fokussiere ich nicht zuletzt auf class, race und gender. Wichtig ist mir hinsichtlich der Argumentation von Biglieri, De Vidovich und Keil (2020), dass die alleinige Fokussierung auf das Periphere tendenziell Dynamiken im Zentrum von Gesellschaft unterschlägt und damit die herrschaftliche Seite der Produktion von Geographien vernachlässigt. Dies birgt die Gefahr, die auf Ungleichheit gerichtete Logik der Peripherisierung zu reproduzieren und aufrechtzuerhalten. Daher werde ich in einem zweiten Schritt die Dimensionen von Zentralität bzw. die Zentralisierung der Geographien der Pandemie beleuchten. Wie wichtig dies ist, darauf verweisen in ihren Repliken auf 
Höhne und Michel mit Blick auf den Globalen Süden Markus Kip (2021) und Marcelo Lopes de Souza (2021), in Bezug auf ländliche Räume in Deutschland Matthias Naumann (2021) und mit Verweis auf Sorgearbeit Anke Strüver (2021). Konkret diskutiere ich vier Dimensionen: Verstädterung, Mobilität, Primat der Wirtschaft und Governance.

Ausgangspunkt für das Argument sind Überlegungen von Henri Lefebvre. Er macht deutlich, dass Räume „Projektion[en] der sozialen Beziehungen“ auf das Terrain sind, sie stets umkämpft bleiben und die verräumlichten Ordnungen daher nicht nur produziert, sondern auch reproduziert werden müssen (Lefebvre 2014 [1970]: 95). Hierbei werden gesellschaftliche Zentralitäten und Peripherien verräumlicht. Keil (2021b: 187) betont, dass „die periphere Position [...] keine fixe quantitative Realität [ist], sondern ein wandelbares Produkt schnell wechselnder Einflüsse und langwieriger Prozesse sozialer Unterdrückung“. Kurzum, die Produktion von Zentralität exkludiert. Das Andere, das Nicht-Machtvolle, wird auf seinen peripheren Platz reduziert (Miggelbrink 2020: 66) und ins „Unvernehmen“ (Rancière 2002 [1995]) verbannt. Ungleichheitsverhältnisse werden so festgeschrieben und Widerspruch gegen diese Zuordnungen wird als Lärm missachtet und übergangen (Rancière 2002 [1995]: 62). Lefebvre folgend, produziert jede Gesellschaftsordnung eigene zentrale Formen der ökonomischen, politischen und sozialen Machtbeziehungen. Für ihn ist Zentralität eine Form, „die an sich leer ist, aber nach Inhalten ruft - nach Objekten, natürlichen oder künstlichen Wesen, Dingen, Produkten und Werken, Zeichen und Symbolen, Menschen, Handlungen, Situationen, praktischen Beziehungen“" (Lefebvre 1991 [1974]: 332; Übers. d. A.). In kapitalistischen Gesellschaften materialisiert sich Zentralität nicht zuletzt in Stadt als Verdichtung von herrschaftlichen und ökonomisch bestimmenden Prozessen. Die Thesen von Höhne und Michel (2021), insbesondere die Annahme eines pandemiebedingten Exodus aus der Stadt, des Zusammenbruchs des Kulturlebens oder Prozessen der Individualisierung tun der dahingehenden Zentralität von Stadt keinen Abbruch. Sie dürften die Geographien von Zentrum und Peripherie aber sehr wohl verschieben, wahrscheinlich zulasten der Prekarisierten.

\section{Geographien der Peripherisierung}

Am Beispiel der Lombardei, Italien, und des Großraums von Toronto, Kanada, diskutieren Biglieri, De Vidovich und Keil (2020) die Auswirkungen von Verstädterung auf das Pandemiegeschehen. Ihr Augenmerk richten sie auf „vergessene Dichten“ (Pitter 2020) - Orte, an denen das Prekariat zusammenkommt, wo Wohnverhältnisse eng sind und Gesundheit aufgrund von Lebenssituationen sowie Mangel an Ressourcen und Zugängen zum Gesundheitswesen auch ohne Covid-19 gefährdet ist. In gewisser Weise schauen sie dorthin, wo Höhne und Michel, wie Hannah Schilling (2021) zu Recht kritisiert, zu wenig hinschauen: auf den „Alltag der Vielen“, der sich anders verändert als jener der eher privilegierten Milieus, die ins Homeoffice wechseln und deren Theater- und Konzertbesuche sowie Städtereisen abgesagt wurden. Bilglieri, De Vidovich und Keil (2020: 3) unterstreichen die Wechselwirkungen zwischen prekären Lebensrealitäten, Peripherisierung und Krankheiten, wobei sie Sorgearbeit als Kulminationspunkt künftiger 
gesellschaftlicher Mobilisierung bestimmen. Anders als SARS im Jahr 2003 (Ali/Keil 2008), das vor allem die Global Cities betroffen habe, durchdringe Covid-19 die verstädterten Landschaften und damit die Gesellschaft als Ganzes. Jedoch sei die Betroffenheit ungleich verteilt. Wo das Virus konzentriert auftrete, dort finde sich das Periphere in der Stadt und in der Gesellschaft, so ihre zentrale These (Biglieri/De Vidovich/Keil 2020: 1).

Keil (2021a; b: 188) bestimmt im Anschluss räumliche, institutionelle und soziale Peripherie als drei relational verwobene Dimensionen. Räumliche Peripherie ist an Orten zu finden, die in der verräumlichten Gesellschaftsordnung nicht als zentral gelten, etwa Großwohnsiedlungen am Stadtrand oder rurale Regionen. Bei der institutionellen Peripherie handelt es sich um behördliche Praxen, Verträge und Gesetzeslagen, die Gesellschaft steuern und Menschen in Sozialwohnungen, Gefängnissen, Flüchtlingslagern, Altenheimen oder dem Niedriglohnsektor einen Platz zuweisen. Soziale Peripherie benennt die rassistische und identitätspolitische Spaltung der Gesellschaft, die dafür sorgt, dass Menschen Teilhabe verwehrt wird. Ich erweitere hier das Argument von Keil und fokussiere auf die intersektionalen Kategorien class, race und gender.

Bezogen auf Deutschland gibt es für keine der drei Dimensionen, anders als für die USA oder Großbritannien, valide Daten (vgl. Wachtler et al. 2020a). So lassen sich hinsichtlich soziodemografischer Faktoren von Covid-19-Erkrankungen lediglich für die Länder Berlin und Bremen Aussagen über Alter und Geschlecht hinaus treffen (Flade et al. 2021). Einige wenige Studien zur räumlichen Peripherie gibt es jedoch (vgl. RKI 2021; Plümper/Neumayer 2020; Wachtler et al. 2020b) - sie verweisen allesamt darauf, dass im Laufe der Pandemie sozialräumlich marginalisierte Regionen immer klarer zu den eigentlichen Hotspots der Pandemie avancierten. Eine Studie des Robert Koch-Instituts (RKI) hat die Covid-19-Sterblichkeit in Relation zur sozioökonomischen Deprivation auf der Ebene von Landkreisen untersucht. Die Autor_innen stellen fest, dass im Winter 2020/21 „die Covid19-Sterblichkeit in sozial stark benachteiligten Regionen um rund 50 bis 70 Prozent höher" lag als in Regionen mit "geringer sozialer Benachteiligung“ (RKI 2021). Die Geographie der sozioökonomischen Deprivation spaltet Deutschland in Ost und West sowie in Nord und Süd, wobei der Osten und der Norden stärker betroffen sind (Wachtler et al. 2020b: 21).

In ihrer räumlichen Analyse der Pandemie arbeiten Nadine Blätgen und Antonia Milbert (2020: 39) heraus, dass das Infektionsgeschehen zwischen März und November 2020 in Städten „leicht höher“ lag als im ländlichen Raum. Auf der Suche nach Ursachen fällt der Blick rasch auf urbane Dichten. Hinsichtlich des Infektionsgeschehens ist Dichte jedoch nicht gleich Dichte (vgl. Pitter 2020): Wesentlich sind Fragen der Verfügbarkeit von und des Zugangs zu Ressourcen und Infrastrukturen (Connolly/Keil/Ali 2021: 253). Für die aktuelle Pandemie betonen Blätgen und Milbert (2020: 43), dass Dichte nur im Zusammenhang mit ,weiteren soziodemografischen Disparitäten“, insbesondere Altersstruktur und sozialer Deprivation, einen statistisch messbaren Effekt auf die geographische Verteilung von Covid-19 hat (vgl. auch Haas/Eckstein 2021). Dies deckt sich mit Befunden aus Berlin, Bremen, Hamburg und Köln, wonach Menschen stärker von Covid-19 betroffen sind, die in Stadtteilen mit hoher Bevölkerungsdichte, niedrigerem 
Durchschnittseinkommen, geringerer Kaufkraft und höherer Armutsquote leben (Flade et al. 2021; Haas/Eckstein 2021; Ismar 2021; Sander 2021). Die drastische Differenz der Inzidenz zwischen dem Villenviertel Köln-Hahnwald, wo sie Ende April 2021 bei Null lag, und der Großwohnsiedlung KölnChorweiler mit 543 Fällen je 100.ooo Einwohner_innen, hat die Debatte um die soziale Selektivität der Pandemie - wenn auch erstaunlich spät - befeuert (Haas/Eckstein 2021; Ismar 2021).

Anhand einer detaillierten Analyse des Umgangs mit Massenausbrüchen in prekarisierten Wohnkomplexen in Göttingen (Mai/Juni 2020) und Berlin (Juni 2020) verdeutlichen Louisa Bäckermann und Peter Birke (2021), dass die räumliche Polarisierung im Laufe der Pandemie politisch eher akzentuiert als bekämpft worden ist. Nach dem ersten ,Lockdown“ wurde im Sommer 2020 „zu einer Strategie der kleinräumigen Kontrolle von Infektionen“ übergegangen (Bäckermann/Birke 2021). In den Fokus rückten Gaststätten, Produktionsstätten der Fleischindustrie, aber auch Schulen, Kitas, Unterkünfte von Geflüchteten, Altenheime und prekarisierte Wohnkomplexe. Hinsichtlich der Wohnorte von weniger Privilegierten beobachten die Autor_innen diskursive Muster, die aus Ghetto-, Problemviertel- und Gefahrengebiet-Narrativen bekannt sind: In einem ersten Schritt wird das Pandemiegeschehen verräumlicht, dann werden dem Raum Attribute wie „migrantisch“, ,,arm“ und „renitent“"zugeschrieben, um schließlich die essentialisierten Räume mitsamt den Menschen zum Problem zu erklären. An den konkreten Beispielen von Göttingen und Berlin ist zu sehen, wie rassistisch aufgeladene Stereotype genutzt werden, um die Gefahr der Pandemie zu peripherisieren und von zentralen Bereichen von Gesellschaft fernzuhalten.

Henning Füller und Iris Dzudzek (2020: 166, 170, 174) verweisen auf eine noch grundlegendere Ebene der Externalisierung. Sie unterstreichen, dass das Virus politisch und administrativ auf eine Naturkatastrophe reduziert und vornehmlich national mittels Sicherheitspraxen bekämpft wird. Auch dies diene dem Zweck, von infrastrukturellen und systemischen Mängeln gesellschaftlicher Regulierung abzulenken. Sie resümieren: „Die eigentliche Krise, die es nicht erst mit der Isolierung eines neuartigen Erregers zu bewältigen gilt, spielt sich auf der Ebene gesellschaftlich gemachter ökonomischer Verhältnisse beziehungsweise von Mensch-Umwelt-Verhältnissen ab.“ (Füller/Dzudzek 2020: 172) Aus diesem Grund ist die soziale Krise nicht mit Mitteln der Pandemiebekämpfung lösbar. Dass nun, wie in Köln, versucht wird, die hohen Inzidenzen in peripherisierten Lagen mittels aufsuchender Impfkampagnen zu bekämpfen, ist begrüßenswert (Ismar 2021), ändert an den grundlegenden Ursachen der hohen Inzidenzen - Armut, Exklusion und Nicht-Repräsentation - jedoch nichts. Hinzu kommt, dass die Kampagnen reichlich spät kommen und eine negative Schlagseite haben: Zum einen sind sie geprägt von einer paternalistischen Ansprache, wenn den Menschen je individuell unterstellt wird, dass sie die offizielle Ansprache nicht verstünden, sie zu wenig mobil und tendenziell impfunwillig seien. Zum anderen diente die Verortung der Pandemie in vermeintliche soziale Brennpunkte, wie Bäckermann und Birke (2021) zeigen, als Strategie der Externalisierung der Gefahr. Diese Strategie funktioniert so lange, bis die peripherisierten Hotspots den Impferfolg in den wohlhabenderen Vierteln infrage stellen. Dies mag ein Grund dafür sein, dass die Debatte um Impfquoten in den 
Siedlungen zu dem Zeitpunkt an Fahrt aufnahm, als die Impfkampagne ebenfalls deutliche Fortschritte machte.

Dimensionen der institutionellen Peripherie sind für Deutschland in vielfältigen Bereichen beschreibbar. Zu nennen sind der Zustand des Sozialund Bildungssystems, der strukturelle Mangel an Investitionen in Infrastrukturen, die Neoliberalisierung des Gesundheitswesens, die Etablierung des Niedriglohnsektors, Gentrifizierungsprozesse bzw. der Abbau von Sozialwohnungen oder die Politik der Flüchtlingslager. In allen Fällen wird Menschen ein Platz zugewiesen und dafür gesorgt, dass sie in der Tendenz dort bleiben. Es sind institutionelle Praxen, die mitverantwortlich dafür sind, dass Menschen in Flüchtlingsunterkünften oder in Einrichtungen für Obdachlose einem erhöhten Risiko, an Covid-19 zu erkranken, ausgesetzt sind. Als eine Form der institutionellen „Heim-Exklusion“, wo Schutz nur ungenügend geleistet wird, ist laut Frank Eckardt (2020: 29) auch die Situation in Alten- und Pflegeheimen zu bezeichnen. Die Zahlen seien auf jeden Fall dramatisch, führt Eckardt Ende 2020 weiter aus - so habe ein Drittel aller Covid-19-Gestorbenen in Heimen gelebt.

Ein weiterer Bereich, in dem institutionelle Peripherisierung deutlich hervortritt, ist der Arbeitsbereich. Gemäß Daten des Statistischen Bundesamts waren im Februar 2021 lediglich 12,8 Prozent der Bruttowertschöpfung unmittelbar von den Anti-Pandemie-Maßnahmen betroffen (Janson 2021). Primär sind dies laut Matthias Janson (2021) die Bereiche „Erziehung und Unterricht, Einzelhandel, Gastgewerbe, Kfz-Handel, Reisebüros und -veranstalter sowie die Kunst-, Kultur- und Erholungsbranche“. In Deutschland gingen knapp 30 Prozent der Berufstätigen ins Homeoffice (Goersch 2020; Alipour et al. 2021) - was die These vom „Ende des Büros“ (Höhne/ Michel 2021: 143) nicht gerade stützt. Rund 25 Prozent mehr Homeoffice wäre möglich, aber 45 Prozent der Berufstätigen müssen ihre Arbeit vor Ort verrichten (Alipour et al. 2021). Zahlen aus den USA belegen die damit verbundene enorme soziale Ungleichheit. So können 61,5 Prozent des einkommensstärksten Viertels der Bevölkerung von zu Hause aus arbeiten, während es im untersten Viertel gerade einmal 9,2 Prozent sind (Gamio 2020). So verteilt sich das Risiko einer Ansteckung sozial selektiv. Für Deutschland hat der NDR Zahlen der Techniker Krankenkasse für das Jahr 2020 veröffentlicht (siehe Abb. 1). Im Schnitt wurden aufgrund einer Covid-19-Erkrankung knapp 500 pro 100.000 Erwerbstätige krankgeschrieben. Mit rund 1.200 Fällen waren Pflegekräfte und Kitaangestellte mehr als doppelt so oft betroffen, in Wissenschaft und Forschung Tätige weniger als halb so oft (200 Fälle) (Deutskens 2021).

Die soziale Peripherie von Covid-19 verläuft entlang von class, race und gender. Die bereits zitierten Zahlen des RKI (2021) sind für die Frage nach class relevant. Zwar lässt die soziale Deprivation einer Region nicht unmittelbar darauf schließen, dass ihre ärmeren Bewohner_innen stärker von Covid-19 betroffen sind. Die Zahlen belegen jedoch, dass Bezieher_innen von Hartz IV nahezu doppelt so oft aufgrund von Covid-19 hospitalisiert werden wie Erwerbstätige. Mitverantwortlich dafür ist, dass ärmere Menschen vielfach Vorerkrankungen haben (Wahrendorf et al. 2021: $317 \mathrm{f}$.). Auch ohne Pandemie haben Frauen mit niedrigem Einkommen eine im Schnitt 4,4 Jahre kürzere Lebenserwartung als Frauen mit hohem Einkommen, bei Männern 


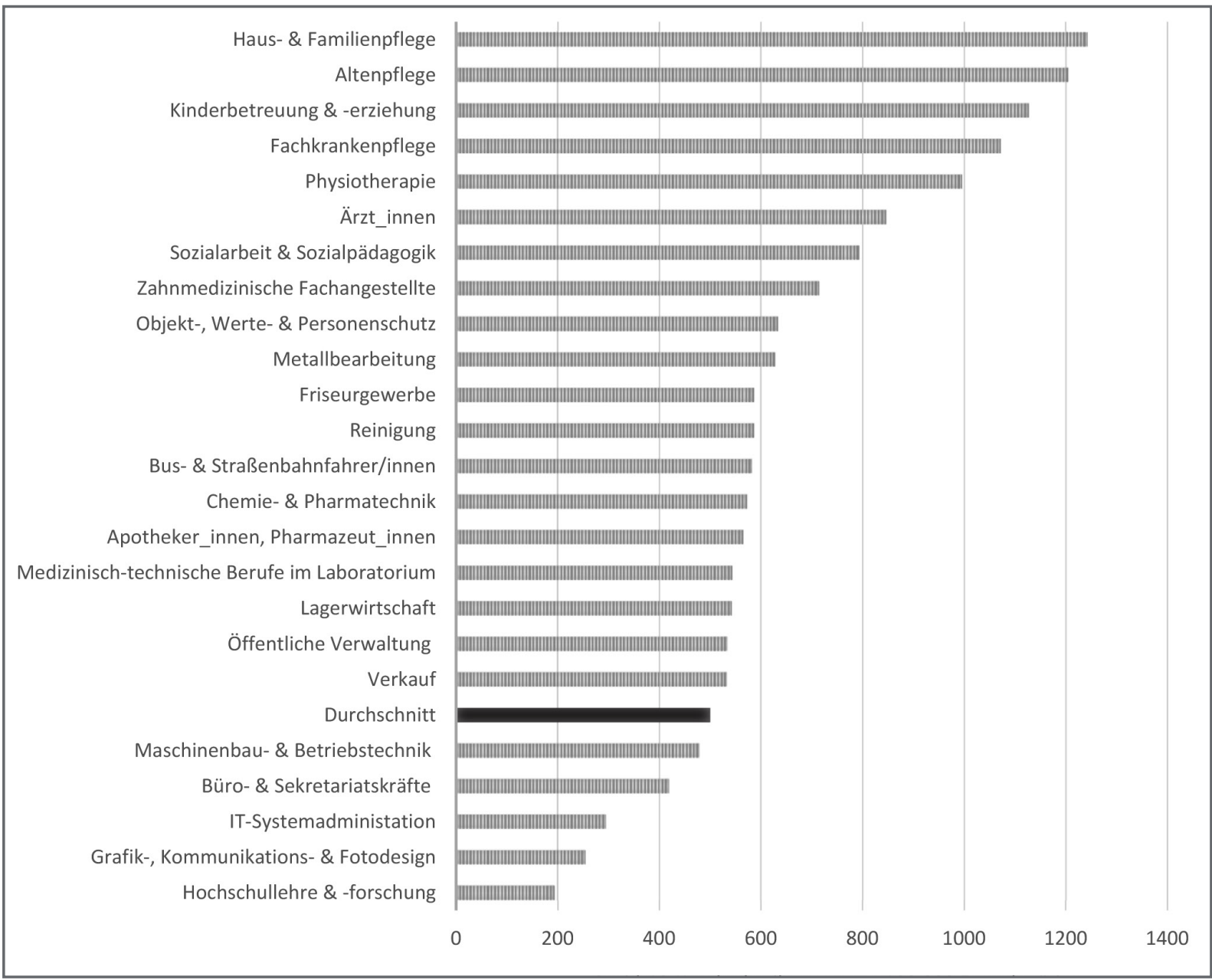

macht die Diskrepanz sogar 8,6 Jahre aus (Wahrendorf et al. 2021: 314). Hinzu kommt, dass prekäre Arbeit meist vor Ort verrichtet werden muss, was dazu führt, dass ärmere Menschen dem Virus stärker ausgesetzt sind. Weniger wohlhabende Menschen leben zudem oftmals in beengten Wohnverhältnissen. $\mathrm{Zu}$ Hause zu lernen oder zu arbeiten, sich gegenseitig aus dem Weg zu gehen, ist kaum möglich. Vielerorts sind auch die notwendigen digitalen Infrastrukturen nicht verfügbar. Der Deutsche Gewerkschaftsbund (DGB 2021: 9 f.) hat zudem herausgestellt, dass die finanziellen Einbußen in der Pandemie vor allem in den unteren Einkommensschichten hoch waren, Abb. 1 Krankschreibungen aufgrund von Covid-19-Diagnosen pro 100.000 Erwerbstätige im Jahr 2020 (Auswahl; eigene Darstellung nach NDR und Techniker Krankenkasse in Deutskens 2021) während Besserverdienende kaum Einbrüche hinnehmen mussten und viele Superreiche ihre Vermögen sogar mehren konnten. Das Alltagsleben der prekären Vielen ist also stark von Covid-19 betroffen, wenngleich darüber weit weniger bekannt ist als über das Leben der Mittel- und Oberschichten in ihrer individualisierten, familiären Häuslichkeit.

Zur Auswirkung von race auf die soziale Peripherie von Covid-19 gibt es für Deutschland kaum Daten. Wenn überhaupt, gibt es Informationen zu Menschen mit Migrationshintergrund. Aus den USA, Großbritannien und Kanada ist bekannt, dass People of Color wesentlich öfter an Covid-19 erkranken und sterben als weiße Menschen (PHE 2020; CDC 2021; Keil 2021a). Das Zusammenwirken von race, prekärer Arbeit, Armut und dem gesteigerten Zugriff durch Sicherheitskräfte in den USA hat Catherine Powell (2020) als „interlocking pandemics“ beschrieben. In den OECD-Staaten erkranken Menschen mit Migrationshintergrund etwa doppelt so oft wie Menschen ohne Migrationshintergrund (OECD, zit. nach Mijnssen 2021). Die Daten aus 
Berlin, Bremen, Hamburg und Köln, nach denen eher prekarisierte Stadtteile von Covid-19 betroffen sind, legen nahe, dass Menschen mit Migrationshintergrund stärker betroffen sind (sieheinsb. Haas/Eckstein 2021 zu Köln). Das liegt daran, dass der Anteil von Menschen mit Migrationshintergrund an ärmeren Bevölkerungsschichten überproportional hoch ist (Metzing 2021: 291). Das erhöhte Risiko von Migrant_innen, an Covid-19 zu erkranken, wird auch mit ihrem eingeschränkten Zugang zum Gesundheitssystem und zu Testzentren, der verbreiteten Nutzung des ÖPNVs, mit der schlechteren Ansprache durch die Versorger_innen, mit bestehenden Kommunikationsbarrieren und dem Unwillen von Behörden erklärt (Filzmaier/Perlot 2021; Haas/Eckstein 2021; Snethlage 2020). So wurde in den großen Geflüchtetenunterkünften noch Mitte April kaum geimpft, obwohl dies seit Ende Februar möglich gewesen wäre (Röhlig 2021). Die globale Ungleichheit des Zugangs zu Vakzinen setzt sich hier im Kleinen fort.

Ein weiterer Ort der Ungleichheit ist der Arbeitsbereich. So ist der Anteil von Menschen mit Migrationshintergrund, die im (systemrelevanten) Niedriglohnsektor arbeiten, weit größer als derjenige von Menschen ohne Migrationsgeschichte. Zahlen von 2018 zufolge haben 28,5 Prozent der abhängig Beschäftigten im Niedriglohnsektor einen Migrationshintergrund, während dieser Anteil in der „autochthonen Bevölkerung“ nur bei 19,5 Prozent liegt (Grabka/Göbler 2020: 24 f.). Hinzu kommt, dass Menschen mit Migrationshintergrund öfter pandemiebedingt entlassen werden (IAB 2020: $11 \mathrm{f}$.).

Wie class und race fällt auch gender als Kategorie der Ungleichheit ins Gewicht. Männer haben zwar häufiger einen schweren Verlauf von Covid-19Erkrankungen und sterben öfter als Frauen. Demgegenüber erkranken Frauen öfter und scheinen vermehrt Langzeiterkrankungen auszuprägen. Die höhere Betroffenheit von Frauen hat ihre Ursache unter anderem in ihrer beruflichen Exponiertheit. In den stark von Covid-19 betroffenen Berufsgruppen (siehe Abb. 1) arbeiten überdurchschnittlich viele Frauen. Gemäß den Daten des Statistischen Bundesamts ist das Personal in Krankenhäusern zu über 75 Prozent weiblich, in der ambulanten Pflege sind es über 8o Prozent (Mullis 2020). In den am wenigsten betroffenen Berufsgruppen liegt der Männeranteil hingegen bei bis zu 86 Prozent (Deutskens 2021).

Mütter tragen zudem angesichts von Schul- und Kitaschließungen die Hauptlast der Kinderbetreuung, wenn auch Väter „überproportional mehr Zeit in die Betreuung ihrer Kinder“ investierten als zuvor (Zinn et al. 2020). Von Geschlechtergerechtigkeit kann noch immer keine Rede sein, so Strüver (2021: 167). Was die Pandemie mit ihrer „Verdichtung der Körper im Wohnraum“ erreicht habe, ist, die „Funktionsmischung auf engstem Raum“ zu radikalisieren. „Dadurch hat die Coronakrise die Carekrise und die anhaltend untertheoretisierten Grundlagen kapitalistischer Gesellschaften, die Sorgearbeit, sicht- und spürbar gemacht." (Strüver 2021: 168) Ebenfalls zugenommen hat in Zeiten der Pandemie die häusliche Gewalt, von der Frauen und Kinder besonders stark betroffen sind. Genaue Zahlen liegen für Deutschland nicht vor. Verschiedene Opferberatungsstellen sprechen für 2020 von einer Zunahme der Meldungen von 8 bis 1o Prozent im Vergleich zum Vorjahr. Berlin (Land Berlin 2021) und Niedersachsen (Niedersächsisches Ministerium für Inneres und Sport 2021) belegen eine Zunahme der häuslichen Gewalt von jeweils rund 7 Prozent im Vergleich zu 2019. Belegt ist 
zudem eine zyklische Dynamik der Fälle mit enormen Ausschlägen während der Schließung von Schulen und Kitas.

Insgesamt, scheint mir, formulieren Biglieri, De Vidovich und Keil (2020) eine wichtige Heuristik, um die verräumlichten Ordnungen der Pandemie zu durchdringen. Auch in Deutschland machen die Geographien von Covid-19 peripherisierte Bereiche der Gesellschaft sichtbar. Menschen in prekären Wohn- und Arbeitssituationen sowie entlang von class, race und gender Diskriminierte erkranken und sterben öfter. Zudem werden Diskriminierungen in vielfacher Weise durch die Schutzmaßnahmen verstärkt (Eckardt 2020). Diese gesellschaftlichen Verortungen nehmen Betroffene auch wahr. Nach der ersten Welle der Pandemie im Sommer 2020 erachtete eine Mehrheit der Bevölkerung den Zusammenhalt in der Gesellschaft als verbessert. Alleinerziehende, Menschen mit Migrationshintergrund, Geringverdienende und Menschen mit einer körperlichen Behinderung bewerten den gesellschaftlichen Zusammenhalt jedoch als schlechter im Vergleich zur Zeit vor der Pandemie (Follmer et al. 2020: 10).

\section{Geographien der Zentralisierung und gesellschaftliche Zentralitäten}

So produktiv die Perspektive auf Peripherisierung auch ist, sie hat den bedeutsamen Mangel, dass sie Dimensionen gesellschaftlicher Zentralität tendenziell vernachlässigt. Die unmittelbar herrschaftliche Seite der Produktion der Geographien von Covid-19 droht, unbeachtet zu bleiben. Mir liegt es fern, die populistische Spaltung zwischen den Eliten bzw. Zentralitäten als Täter_innen und der unschuldigen Bevölkerung bzw. den Peripherien zu befördern. Michel Foucault (1982: 240-247) macht unmissverständlich klar, dass das Subjekt immer Teil der bestehenden Verhältnisse ist, sie hervorbringt und reproduziert. Gleichzeitig belegen Grégoire Chamayou (2019) und Jamie Peck (2008), dass die gegenwärtige neoliberalisierte Gesellschaftsordnung auf politischen Strategien und der (autoritären) Durchsetzung im ökonomischen und politischen Zentrum der Gesellschaft beruht. Ich werde im Folgenden vier Bereiche skizzieren, in denen Zentralität bzw. Prozesse der Zentralisierung für Geographien der Pandemie von Bedeutung sind: Verstädterung, Mobilität, Primat der Wirtschaft und Governance. Die Überlegungen sind nicht abschließend, dürften aber wichtige Punkte abdecken. Dennoch kommen in der Art und Weise, wie die Pandemie konfrontiert wird, auch andere Zentralitäten zum Vorschein, etwa das Primat der Kleinfamilie und des Eigenheims mit Garten. Ich formuliere meine Überlegungen zur Zentralität nicht als Widerspruch zur Perspektive auf Peripherisierung (Biglieri/ De Vidovich/Keil 2020), sondern es geht mir eher darum, Hinweise auf die Wechselverhältnisse zwischen Zentralität bzw. Zentralisierung und Peripherie bzw. Peripherisierung in der Produktion der Geographien von Covid-19 zu geben (vgl. Naumann 2021).

Erstens: Verstädterung. Die Lombardei und der Großraum von Toronto dienen Biglieri, De Vidovich und Keil (2020) in ihren Ausführungen zu den Auswirkungen von Verstädterung auf das Pandemiegeschehen als Ausgangspunkte. Beide Regionen sind, global betrachtet, hoch urbanisiert und zentralisiert - es handelt sich um zwei wirtschaftlich prosperierende 
Räume in G7-Staaten. Gerade die Maßstabsebene der Betrachtung spielt für die Bewertung von Zentralität und Peripherie offenkundig eine wichtige Rolle. Es macht also einen bedeutsamen Unterschied, ob die Geographien aus einer globalen oder einer städtischen Perspektive betrachtet werden (Smith 1987: 64). Mit Blick auf den Globalen Süden unterstreichen Kip (2021) und de Souza (2021), bezogen auf ländliche Räume in Deutschland betont Naumann (2021), dass Peripherie nicht gleich Peripherie ist. Zwar betrifft überall - von Bengaluru und Mohali über Beirut, Tunis und Berlin bis zu Mexiko-Stadt, Nairobi und Johannesburg - die „Pandemie ohnehin schon sozioökonomisch benachteiligte Gruppen“ weit stärker (Kip 2021: 172). Die Betroffenheit in peripheren Regionen des Amazonas oder den Townships von Johannesburg ist jedoch eine andere als in den ländlichen Peripherien Deutschlands oder den Vororten von Toronto und Mailand. Womöglich haben hier die von Maximilian Förtner, Bernd Belina und Matthias Naumann (2019) eingeführten Begriffe umfassende Peripherisierung, peripheres Zentrum sowie zentrale Peripherie ein Potenzial zur Weiterentwicklung.

Neben anderen hat Keil selbst die zentrale Bedeutung der extensiven Urbanisierung von Gesellschaft für die Entwicklung, die Ausbreitung und die Bekämpfung von Pandemien hervorgehoben (vgl. Ali/Keil 2008; Connolly/ Keil/Ali 2021; Davis 2020 [2005]). Die Genese des Virus und seine gesellschaftliche Affizierung hängen somit auf globaler Ebene ganz grundsätzlich mit Prozessen kapitalistischer Zentralisierung und urbanisierter MenschNatur-Verhältnisse zusammen. Wenn auch Höhne und Michel (2021: 142) zuzustimmen ist, dass Covid-19 zu ,tiefgreifenden Transformationen nahezu jeden Aspekts des urbanen Lebens geführt“ hat, geht in Anbetracht der globalen Wirkmacht von Verstädterungsprozessen die These der „Enturbanisierung" mit Sicherheit zu weit. Pointiert antwortet Anna-Lisa Müller (2021): „Kein Ende, sondern eine Veränderung des Städtischen“. Sie betont die digitale „Neuformierung sozialer Interaktionen“ sowie die „Restrukturierung des öffentlichen Raums“" (Müller 2021: 201). Ich möchte jedoch unterstreichen, dass Veränderung als Perspektive der tendenziellen gleichförmigen Verschiebung zu kurz greift. Covid-19 wird zu einer weiteren Akzentuierung sozialer Polarisierung in den urbanisierten Landschaften führen und damit soziale Krisen nicht nur verändern, sondern vertiefen. Während sich der weit kleinere Teil der urbanen Bevölkerung verstärkt „sichere Orte“ mit Platz und Grün erschließen und absichern wird, dürften die Vielen eher in die vergessenen Dichten und damit an Orte gedrängt werden, die mit „Überfüllung, Enge, Chaos und Krankheit“ (Roskamm 2021: 206) konnotiert sind. Zu befürchten ist eine Art Rückkehr der bürgerlichen Angst vor urbanisierter Dichte mit allen negativen Folgen der sozialen und paternalistischen Kontrolle und Exklusion (vgl. Lindner 2007; Roskamm 2021).

Zweitens: Mobilität. Der zentrale Mechanismus der von Wuhan, China, ausgehenden Ausbreitung von Covid-19 war der globale Flugverkehr, also Mobilität (Mouton et al. 2020). Dass sich auch hier bedeutsame Relationen zwischen Zentrum und Peripherie beschreiben lassen, darauf verweisen Daten hinsichtlich der Verbreitung von Covid-19, die mir Dieter Müller von medico international zur Verfügung gestellt hat: So dürfte der erste bestätigte Covid-19-Fall in Südafrika auf einen Skiurlaub in Italien zurückgehen (NICD 2020) und die erste Infektion im Senegal auf einen französischen 
Staatsbürger, der aus Frankreich zurückkehrte (WHO 2020). Die erste Covid19-Tote in Brasilien war eine Haushaltshilfe, die sich bei ihrer Arbeitgeberin angesteckt hatte, die sich ihrerseits beim Karneval in Italien infiziert haben dürfte (Blasberg 2020). In gewisser Weise wurde das Virus von lokalen Eliten, die sich Reisen in den Globalen Norden leisten können, ins Land gebracht, bevor es sich mit voller Wucht in den gesellschaftlichen Peripherien ausbreitete. Aber auch Arbeitsmigration zwischen Zentrum und Peripherie sowie transnationale Warenketten sind von Bedeutung. So geht einer der ersten Fälle in Ecuador auf eine ecuadorianische Staatsbürgerin zurück, die in Spanien gearbeitet, aufgrund der Krise jedoch arbeitslos geworden und zurückgekehrt war (España 2020). In Indien trug die massenhafte Bewegung aus den urbanen Zentren in die Peripherien zur Ausbreitung des Virus bei. Als während der ersten Welle von März bis September 2020 ein Lockdown verhängt wurde, strömten die prekarisierten Arbeiter_innen arbeitslos zurück in die Peripherie (Verma 2020). In Deutschland stand der erste bestätigte Fall in enger Beziehung zu internationalisierten Produktionsketten der Autoindustrie. Am Hauptsitz eines Oberbayerischen Autozulieferers, der in Wuhan ein Werk betreibt, fand eine Schulung statt, an der eine Mitarbeiterin aus China teilnahm (Kunkel 2021).

Diese globalen Verknüpfungen zusammen mit dem Faktor Skiurlaub in Italien und Österreich haben in Deutschland dafür gesorgt, dass die ersten Hotspots der Pandemie in den wirtschaftlich starken und wohlhabenden Regionen von Bayern, Baden-Württemberg und Hamburg auftraten (Blätgen/Milbert 2020: 34). Erst mit der Zeit, so belegen Studien (Plümper/ Neumayer 2020; Wachtler et al. 2020b), verschob sich der Schwerpunkt des Pandemiegeschehens in Regionen mit erhöhter sozioökonomischer Deprivation. Deutlich wird: Globale Mobilität hat viel mit Mustern der Ungleichheiten zwischen Zentrum und Peripherie zu tun und diese Ungleichheiten sind relevant für die Ausbreitung des Virus und wo es verdichtet.

Drittens: Primat der Wirtschaft. Die Fokussierung auf Prozesse der Peripherisierung kaschiert an mancher Stelle, dass das Pandemiegeschehen Ausdruck der Zentralität eines Sektors sein kann. Die hohen Zahlen von erkrankten, meist weiblichen Pflegekräften sind nicht allein Ausdruck für deren Peripherisierung, sondern deutliches Zeichen der zentralen Relevanz des Gesundheitssektors in der Pandemie. Das Argument lässt sich unter anderen Vorzeichen für die Ausbrüche in der Fleischindustrie, in Logistikzentren sowie Kitas und Schulen fortsetzen. Im ersten Fall trafen die hohen Inzidenzen eine bedeutsame Exportindustrie, die in manchen Regionen Deutschlands die Wertschöpfungsketten bestimmt und somit nicht stillstehen durfte (Birke 2021). Für den zweiten Fall beschreibt Agnieszka Mróz (2021), Amazon-Arbeiterin im polnischen Poznań, exemplarisch die Rolle ihrer Sparte sowie ihre eigene Position darin: „Wir sind keine Opfer, wir arbeiten an einer zentralen Schaltstelle des globalen Kapitalismus, die für das Funktionieren des Warenkreislaufs entscheidend ist." Und im dritten Fall wird trotz der Verweise auf Bildungsgerechtigkeit und Chancengleichheit deutlich, dass Schulen und Kitas vor allem deshalb offenbleiben bzw. geöffnet wurden, um die Arbeitskraft der Eltern verfügbar zu halten. Auch das nun von der Bundesregierung vorgelegte „Aufholprogramm“ adressiert die pandemiebedingten Entbehrungen von Kindern und Jugendlichen vornehmlich 
als Bildungsdefizite, die eine Gefahr für den Wirtschaftsstandort Deutschland darstellen (Brandes 2021; Schnaas 2021).

In Deutschland wurden während der langen Monate der Pandemie zentrale Bereiche der Wirtschaft und Industrie geschützt und die Produktion wurde aufrechterhalten. Wie umfassend dies umgesetzt wurde, verdeutlichen die oben zitierten Zahlen: 87,2 Prozent der Bruttowertschöpfung waren im Februar 2021 nicht unmittelbar von den Maßnahmen zur Bekämpfung der Pandemie betroffen (Janson 2021). Anstatt den Schutz der Menschen zu erhöhen, wurden in einigen strategisch relevanten Sektoren, etwa in der Pflege, Qualitätsstandards aufgeweicht (ntv 2020) und Arbeitszeitenregelungen gelockert (Malburg 2020). Gleiches gilt für landwirtschaftliche Erntehelfer_ innen, die meist für die Saisonarbeit aus Osteuropa kommen. Hier wurde die Dauer einer sozialversicherungsfreien Beschäftigung von 70 auf 102 Tage erhöht; in Fällen von Massenausbrüchen wurde „Arbeitsquarantäne“ verhängt (Strack 2021).

Viertens: Governance. Der politische Umgang mit der Pandemie ist in Deutschland von Bundesland zu Bundesland und global gesehen von Land zu Land (sehr) unterschiedlich. Die Reaktionen in Demokratien wie Diktaturen reichen von Verharmlosung und Leugnung bis hin zu drastischen Lockdowns, schwerwiegenden Eingriffen in die Grundrechte und zur Intensivierung von Überwachung und Kontrolle. Der Ort der politischen Entscheidung ist nicht per se der Nationalstaat und im Laufe der Pandemie waren skalare Verschiebungen der politischen Regulierung zu beobachten. In Frankreich etwa nimmt der Nationalstaat unbestritten die zentrale Rolle ein, während im föderalen Deutschland die Bekämpfung der Pandemie vornehmlich Ländersache oder gar eine Angelegenheit der Kommunen ist. Mit der „Bundes-Notbremse“ vom April 2021 wurde in Deutschland hingegen eine Reskalierung von Zentralität auf die Ebene des Nationalstaates vorgenommen. In Indien ging Premierminister Narendra Modi einen anderen Weg. Während er in der ersten Welle die Pandemie auf nationaler Ebene regulierte, delegierte er die Lockdowns in der zweiten Welle im Frühjahr 2021 auf die Ebene der Bundesstaaten, um nicht weiterhin für unpopuläre Entscheidungen verantwortlich zeichnen zu müssen (SRF 2020). Wichtig scheint mir also mit Blick auf die Geographien von Covid-19, dass die administrative Regulierung der Pandemie - auf welcher Maßstabsebene auch immer sie vollzogen wird - eine Form der Zentralität darstellt, die für die Produktion von sicheren und unsicheren Orten relevant ist (vgl. Collard 2012; Hannah et al. 2020). Besonders deutlich wird dies mit Blick auf die nationale Ebene. So hat etwa das ignorante Agieren nationaler Behörden - um Donald Trump in den USA, Jair Bolsonaro in Brasilien oder Narendra Modi in Indien - viel mit den dramatischen Verläufen der Pandemie in den jeweiligen Ländern zu tun. Die oben beschriebenen Prozesse der Peripherisierung haben aber immer auch eine Dimension der politischen Produktion und werden daher an Orten der politischen Zentralität hervorgebracht.

\section{Schluss}

Die Geographien von Covid-19 markieren auch in Deutschland vielfältige räumliche, institutionelle und soziale Peripherisierungen, wobei die 
Ungleichheiten entlang von class, race und gender verlaufen. Die Perspektive von Biglieri, De Vidovich und Keil (2020) erweist sich als enorm hilfreich, um die Ungleichheiten klar zu erfassen. Die Fokussierung auf Peripherisierung reicht jedoch nicht aus, um die Geographien von Covid-19 zu entschlüsseln. Mir scheint, dass mit Zentralität assoziierte Prozesse der globalen Urbanisierung, das Primat der Wirtschaft und ihrer Netzwerke sowie Regierungsweisen eine bedeutsame Rolle für die Ausbreitungsmuster des Virus haben und somit bedacht werden müssen. Die Bedeutung von Zentralität zu betonen, heißt jedoch nicht, zu behaupten, dass die Last des Virus nicht vor allem in den Peripherien geschultert würde. Peripherisierte Menschen in marginalisierten Regionen sind öfter und stärker von Krankheit und Entbehrungen betroffen als Menschen in den Zentren der Gesellschaft. Peripherie ist nicht gleich Peripherie. Die Maßstabsebene der Betrachtung ist relevant dafür, was als solche in Erscheinung tritt. Peripherisierung ist ein politischer Prozess, der Menschen einen Platz zuweist. Diese Plätze können auf einer anderen Ebene zentral sein, etwa die Arbeit im Gesundheitswesen, in Logistikzentren oder Schulen und Kitas. Die Formierung der Subjekte als peripher in den zentralen „Schaltstellen des globalen Kapitalismus“(Mróz 2021) ist eine Praxis des Unvernehmen-Machens der Stimmen. Sie dient einerseits der Externalisierung von sozialen Problemlagen auf bestimmte Gruppen mit zugeschriebenen Merkmalen entlang von class, race und gender sowie dem Verfügbarhalten billiger Arbeitskräfte. Die alleinige Fokussierung auf Peripherien und die damit einhergehende Zuschreibung von peripheren Positionen birgt daher die Gefahr, die Ordnung der Ungleichheit zu reproduzieren und damit (ungewollt) zu stützen. Deshalb ist das Betonen von Zentralitäten der Pandemie unerlässlich. Covid-19 markiert somit nicht allein die Peripherie in der Stadt und der Gesellschaft, sondern auch gut kaschierte Zentralitäten kapitalistischer Vergesellschaftung. Die Pandemie macht in gewisser Weise das Unsichtbare sichtbar (Haid 2021). Darin liegt ein politisches Potenzial zum gemeinsamen Erringen des „Rechtes auf Zentralität“ (Lefebvre 2014 [1970]: 144) und einer wirklich urbanen Gesellschaft.

Dieser Beitrag wurde veröffentlicht mit Unterstützung des Open-AccessPublikationsfonds der Leibniz-Gemeinschaft.

\section{Endnoten}

[1] Ich bedanke mich bei Louisa Bäckermann, Peter Birke sowie der s u b \u r b a n-Redaktion für die aufmerksame Lektüre und die hilfreichen Kommentare.

\section{Autor_innen}

Daniel Mullis ist Humangeograph. Er forscht zu politischer Philosophie, Sozialprotesten sowie Krisendynamiken, aktuell insbesondere zum Neuen Autoritarismus. mullis@hsfk.de 


\section{Literatur}

Ali, Harris S. / Keil, Roger (Hg.) (2008): Networked disease. Emerging infections in the global city. Malden: Wiley-Blackwell.

Alipour, Jean-Victor / Falck, Oliver / Peichl, Andreas / Sauer, Stefan (2021): HomeofficePotenzial weiterhin nicht ausgeschöpft. München: ifo Institut.

Bäckermann, Louisa / Birke, Peter (2021): Über die stadtpolitische Lokalisierung der Corona-Krise in Göttingen und Berlin. https://coronamonitor.noblogs.org/2021/01/15/ gefaehrliche_orte/ (letzter Zugriff am 13.4.2021).

Biglieri, Samantha / De Vidovich, Lorenzo / Keil, Roger (2020): City as the core of contagion? Repositioning Covid-19 at the social and spatial periphery of urban society. In: Cities \& Health. https://doi.org/10.1080/23748834.2020.1788320.

Birke, Peter (2021): Die Fleischindustrie in der Coronakrise. Eine Studie zu Arbeit, Migration und multipler Prekarität. In: Sozial.Geschichte Online 29, 1-47.

Blasberg, Marian (2020): Stehpinkler und ihre Mägde. In: Der Spiegel 22/2020, 86-92.

Blätgen, Nadine / Milbert, Antonia (2020): Dichte und Pandemie - Gibt es räumliche Erklärungsmuster für das Infektionsgeschehen in Deutschland? In: Informationen zur Raumentwicklung 47/4, 30-45.

Brandes, Tanja (2021): Jugend in der Pandemie: Entweder effizient - oder verloren. In: Berliner Zeitung, 11.5.2021. https://www.berliner-zeitung.de/politik-gesellschaft/ jugend-in-der-pandemie-entweder-effizient-oder-verloren-li.158339 (letzter Zugriff am 25.5.2021).

Brenner, Neil / Schmid, Christian (2015): Towards a new epistemology of the urban? In: City 19/2-3, 151-182.

CDC (2021): Risk for Covid-19 infection, hospitalization, and death by race/ethnicity. Centers for Disease Control and Prevention. https://www.cdc.gov/coronavirus/2019ncov/Covid-data/investigations-discovery/hospitalization-death-by-race-ethnicity.html (letzter Zugriff am 13.4.2021).

Chamayou, Grégoire (2019): Die unregierbare Gesellschaft. Eine Genealogie des autoritären Liberalismus. Berlin: Suhrkamp.

Collard, Rosemary-Claire (2012): Cougar - Human entanglements and the biopolitical un/making of safe space. In: Environment and Planning D 30/1, 23-42.

Connolly, Creighton / Keil, Roger / Ali, Harris S. (2021): Extended urbanisation and the spatialities of infectious disease. Demographic change, infrastructure and governance. In: Urban Studies 58/2, 245-263.

Davis, Mike (2020 [2005]): Vogelgrippe. Zur gesellschaftlichen Produktion von Epidemien. Pdf-Version. Berlin/Hamburg: Assoziation A.

Deutskens, Annette (2021): Pflegekräfte und Kita-Beschäftigte erkranken öfter an Covid. In: NDR, 7.3.2021. https://www.ndr.de/nachrichten/niedersachsen/Pflegekraefte-undKita-Beschaeftigte-erkranken-oefter-an-Covid,corona7020.html (letzter Zugriff am 13.4.2021).

DGB (2021): Verteilungsbericht 2021. Berlin: Deutscher Gewerkschaftsbund Bundesvorstand.

Eckardt, Frank (2020): Vertiefung der Gräben. Corona in der fragmentierten Stadt. In: dérive 80, 26-30.

España, Sara (2020): Una mujer llegada de España, primer caso de coronavirus en Ecuador. In: El País, 29.2.2020. https://elpais.com/sociedad/2020-02-29/una-mujer-llegada-deespana-primer-caso-de-coronavirus-en-ecuador.html (letzter Zugriff am 6.5.2021).

Filzmaier, Peter / Perlot, Flooh (2021): Mediennutzung in der Corona-Pandemie. Informationsverhalten von Personen mit Migrationshintergrund zum Thema Corona. Wien: Österreichischer Integrationsfonds.

Flade, Florian / Ghassim, Armin / Grill, Markus / Reisin, Andrej / Schreijaeg, Jonas (2021): Die Ahnungslosigkeit der Politik. In: Tagesschau, 4.3.2021. https://www.tagesschau.de/ investigativ/panorama/corona-soziale-faktoren-101.html (letzter Zugriff am 13.4.2021).

Follmer, Robert / Brand, Thorsten / Unzicker, Kai (2020): Gesellschaftlicher Zusammenhalt in Deutschland 2020. Gütersloh: Bertelsmann Stiftung.

Förtner, Maximilian / Belina, Bernd / Naumann, Matthias (2019): Stadt, Land, AfD. Zur Produktion des Urbanen und des Ruralen im Prozess der Urbanisierung. In: sub $\backslash$ urban. zeitschrift für kritische stadtforschung 7/1-2, 23-44. 
Foucault, Michel (1982): Subjekt und Macht. In: Ders. (2005): Analytik der Macht. Frankfurt am Main: Suhrkamp, 240-263.

Füller, Henning / Dzudzek, Iris (2020): Die Logik des Ausbruchs. Formierung von Covid-19 durch Krisenbearbeitungsweisen. In: sub \urban. zeitschrift für kritische stadtforschung $8 / 3,165-182$.

Gamio, Lazaro (2020): The workers who face the greatest coronavirus risk. In: The New York Times, 15·3.2020. https://www.nytimes.com/interactive/2020/03/15/business/ economy/coronavirus-worker-risk.html?smid=tw-share (letzter Zugriff am 13.4.2021).

Goersch, Henning (2020): Akkon-Bevölkerungsstudien zu Verhalten, Erleben und Bewältigung der deutschen Bevölkerung in der Corona-Krise. Berlin: akkkon Hochschule für Humanwissenschaften.

Grabka, Markus M./ Göbler, Konstantin (2020): Der Niedriglohnsektor in Deutschland. Falle oder Sprungbrett für Beschäftigte? Gütersloh: Bertelsmann Stiftung.

Haas, Simon / Eckstein, Charlotte (2021): Warum Migranten und AfD-Wähler ein höheres Ansteckungsrisiko haben. In: NZZ, 16.5.2021. https://www.nzz.ch/international/ deutschland/warum-corona-migranten-und-afd-waehler-oefter-trifft-ld.1624457 (letzter Zugriff am 25.5.2021).

Haid, Christian (2021): Die Stadt am Ende? Am Ende die Stadt. Chancen für eine gerechtere Stadt mit und nach Covid-19. Kommentar zu Stefan Höhne und Boris Michel. In: sub $\backslash$ urban. zeitschrift für kritische stadtforschung 9/1-2, 193-200.

Hannah, Matthew G. / Hutta, Jan S. / Schemann, Christoph (2020): Thinking through Covid-19 responses with Foucault - an initial overview. In: Antipode Online, 5.5.2020. https://antipodeonline.org/2020/05/05/thinking-through-covid-19-responses-withfoucault/ (letzter Zugriff am 6.4.2021).

Höhne, Stefan / Michel, Boris (2021): Das Ende des Städtischen? Pandemie, Digitalisierung und planetarische Enturbanisierung. In: sub \urban. zeitschrift für kritische stadtforschung 9/1-2, 141-149.

IAB (2020): Befunde der IAB-forschung zur Corona-Krise - Zwischenbilanz und Ausblick. Institut für Arbeitsmarkt- und Berufsforschung 26.8.2020. http://doku.iab.de/ grauepap/2020/IAB-Befunde_Covid-19.pdf (letzter Zugriff am 10.5.2021).

Ismar, Georg (2021): „Der Fehler ist doch: Man hätte nicht jahrzehntelang zündeln dürfen“. Die Corona-Spaltung am Beispiel Köln. In: Tagesspiegel, 3.5.2021. https://www.tagesspiegel.de/politik/die-corona-spaltung-am-beispiel-koeln-der-fehler-ist-doch-manhaette-nicht-jahrzehntelang-zuendeln-duerfen/27149524.html (letzter Zugriff am 4.5.2021).

Janson, Matthias (2021): 13 Prozent der Wirtschaft direkt von Lockdown betroffen. https://de.statista.com/infografik/amp/24068/anteile-der-wirtschaftsbereiche-an-dergesamten-bruttowertschoepfung-deutschlands/ (letzter Zugriff am 13.4.2021).

Keil, Roger (2018): Suburban planet. Making the world urban from outside in. Cambridge/ Medford, MA: Polity Press.

Keil, Roger (2021a): Covid Urban. Dimensionen der Peripherie in der städtischen Gesellschaft, Vortrag am 26.1.2021 im Rahmen der translokalen Vorlesungsreihe „Geographien von Covid-19“. https://vimeo.com/505594604 (letzter Zugriff am 13.4.2021).

Keil, Roger (2021b): Höher, weiter, breiter. Die endlose Stadt nach Covid-19. Kommentar zu Stefan Höhne und Boris Michel. In: sub \urban. zeitschrift für kritische stadtforschung 9/1-2, 185-191.

Kip, Markus (2021): Krisendiagnostik einer kritischen Stadtforschung. Kommentar zu Stefan Höhne und Boris Michel. In: sub \urban. zeitschrift für kritische stadtforschung 9/1-2, 171-177.

Kunkel, Ulrike (2021): Ein Jahr Corona in Deutschland. Wie das Virus zu uns kam. In: ZDF heute, 27.1.2021. https://www.zdf.de/nachrichten/panorama/corona-bayern-chinawebasto-10o.html (letzter Zugriff am 6.5.2021).

Land Berlin (2021): Entwicklung der häuslichen Gewalt 2020. Pressemitteilung vom 3.3.2021. https://www.berlin.de/sen/justva/presse/pressemitteilungen/2021/pressemitteilung.1059664.php (letzter Zugriff am 6.5.2021).

Lefebvre, Henri (1991 [1974]): The production of space. Oxford: Blackwell.

Lefebvre, Henri (2014 [1970]): Die Revolution der Städte. Hamburg: Europäische Verlagsanstalt.

Lindner, Rolf (2007): Ganz unten. Ein Kapitel der Geschichte der Stadtforschung. In: Wien Museum (Hg.): Ganz unten. Die Entdeckung des Elends. Wien: Christian Brandstätter Verlag, 9-17. 
Malburg, Mascha (2020): Ausgequetscht wie Zitronen. In: Neues Deutschland, 9.4.2020. https://www.neues-deutschland.de/artikel/1135340.hubertus-heil-ausgequetscht-wiezitronen.html (letzter Zugriff am 6.5.2021).

Metzing, Maria (2021): Lebenssituation von Migrantinnen und Migranten, deren Nachkommen und Geflüchteten in Deutschland. In: Destatis / WZB / BiB (Hg.): Datenreport 2021. Bonn: bpb, 286-294.

Miggelbrink, Judith (2020): Ländliche Räume - strukturschwach, peripher, abgehängt? In: Krajewski, Christian / Wiegandt, Claus-Christian (Hg.): Land in Sicht. Ländliche Räume in Deutschland zwischen Prosperität und Peripherisierung. Bonn: bpb, 64-79.

Mijnssen, Ivo (2021): Migranten sind in Österreich anfälliger für eine Corona-Erkrankung dies liegt weniger an ihrer Kultur als an der gesellschaftlichen Position. In: Neue Zürcher Zeitung, 6.4.2021. https://www.nzz.ch/international/coronavirus-oesterreich-migrationals-risikofaktor-ld.1609783 (letzter Zugriff am 13.4.2021).

Mouton, Christopher / Grissom, Adam / Godges, John / Hanson, Russell (2020): COVID-19 air traffic visualization. RAND Corporation. https://doi.org/10.7249/RRA248-6 (letzter Zugriff am 19.4.2021).

Mróz, Agnieszka (2021): „Wenn wir wollen, steht das alles still“. In: WOZ 15/2021. https:// www.woz.ch/-b65d (letzter Zugriff am 16.4.2021).

Mullis, Daniel (2020): Mit der Corona-Krise in eine autoritär-individualistische Zukunft? Fünf Dimensionen gesellschaftlicher Transformation. In: PRIF Blog, 21.4.2020. https:// blog.prif.org/2020/04/21/mit-der-corona-krise-in-eine-autoritaer-individualistischezukunft-fuenf-dimensionen-gesellschaftlicher-transformation/ (letzter Zugriff am 10.5.2021).

Müller, Anna-Lisa (2021): Kein Ende, sondern eine Veränderung des Städtischen. Kommentar zu Stefan Höhne und Boris Michel. In: sub\urban. zeitschrift für kritische stadtforschung 9/1-2, 201-204.

Naumann, Matthias (2021): Das Ende des Ländlichen? Covid-19 als Krise ländlicher Räume. Kommentar zu Stefan Höhne und Boris Michel. In: sub\urban. zeitschrift für kritische stadtforschung 9/1-2, 159-164.

NICD (2020): First case of COVID-19 coronavirus reported in SA. The National Institute for Communicable Diseases, 5.3.2020. https://www.nicd.ac.za/first-case-of-covid-19coronavirus-reported-in-sa (letzter Zugriff am 4.5.2021).

Niedersächsisches Ministerium für Inneres und Sport (2021): Polizeiliche Kriminalstatistik 2020: Historisch niedrige Kriminalitätszahlen und gleichzeitig höchste Aufklärungsquote. https://www.mi.niedersachsen.de/startseite/aktuelles/presseinformationen/ polizeiliche-kriminalstatistik-2020-historisch-niedrige-kriminalitatszahlen-und-gleichzeitig-hochste-aufklarungsquote-198676.html (letzter Zugriff am 6.5.2021).

ntv (2020): Pflege-TÜV wird wegen Corona-Krise ausgesetzt. In: ntv, 19.3.2020. https:// www.n-tv.de/ticker/Pflege-TUV-wird-wegen-Corona-Krise-ausgesetzt-article21655060. html (letzter Zugriff am 6.5.2021).

Peck, Jamie (2008): Remaking laissez-faire. In: Progress in Human Geography 32/1, 3-43.

PHE (2020): Disparities in the risk and outcomes of Covid-19. London: Public Health England.

Pitter, Jay (2020): Urban density: Confronting the distance between desire and disparity. https://www.azuremagazine.com/article/urban-density-confronting-the-distancebetween-desire-and-disparity/ (letzter Zugriff am 13.4.2021).

Plümper, Thomas / Neumayer, Eric (2020): The pandemic predominantly hits poor neighbourhoods? SARS-CoV-2 infections and Covid-19 fatalities in German districts. In: European Journal of Public Health 30/6, 1176-1180.

Powell, Catherine (2020): Interlocking Pandemics. In: Dies.: Proceedings of the ASIL Annual Meeting 114, 371-373.

Rancière, Jacques (2002 [1995]): Das Unvernehmen. Politik und Philosophie. Frankfurt am Main: Suhrkamp.

RKI (2021): Soziale Unterschiede in der Covid-19-Sterblichkeit während der zweiten Infektionswelle in Deutschland. Robert Koch-Institut. https://www.rki.de/DE/Content/ GesundAZ/S/Sozialer_Status_Ungleichheit/Faktenblatt_COVID-19-Sterblichkeit.html (letzter Zugriff am 13.4.2021).

Röhlig, Marc (2021): „Das rächt sich in der Pandemie“. In: Spiegel Online, 11.4.2021. https:// www.spiegel.de/politik/deutschland/impfen-fuer-fluechtlingen-verzoegerungen-beimstart-der-impfkampagne-a-46a96oda-74cc-4910-ab8a-3764afd2ed6b (letzter Zugriff am 13.4.2021). 
Roskamm, Nikolai (2021): Urbanistische Heimsuchungen. Kommentar zu Stefan Höhne und Boris Michel. In: sub \urban. zeitschrift für kritische stadtforschung 9/1-2, 205-211.

Sander, Heiko (2021): Corona-Zahlen: Erhebliche Unterschiede in Hamburgs Stadtteilen. In: NDR, 7.4.2021. https://www.ndr.de/nachrichten/hamburg/Corona-Zahlen-Erhebliche-Unterschiede-in-Hamburgs-Stadtteilen,stadtteile628.html (letzter Zugriff am 10.5.2021).

Schilling, Hannah (2021): Lokale urbane Welten sichtbar machen. Kommentar zu Stefan Höhne und Boris Michel. In: sub \urban. zeitschrift für kritische stadtforschung 9/1-2, 179-184.

Schnaas, Dieter (2021): Schule und Corona: Die verachtete Jugend. In: WirtschaftsWoche, 9.5.2021. https://www.wiwo.de/politik/deutschland/tauchsieder-die-verachtete-jugend/ 27172164-all.html (letzter Zugriff am 10.5.2021).

Smith, Neil (1987): Dangers of the empirical turn. Some comments on the CURS initiative. In: Antipode 19/1, 59-68.

Snethlage, Matej (2020): Das Virus ist nicht egalitär. In: taz, 5.5.2020. https://taz.de/ Rassismus-im-Gesundheitswesen/!5679885/ (letzter Zugriff am 13.4.2021).

de Souza, Marcelo Lopes (2021): Die Covid-19-Pandemie bedeutet nicht das Ende des Städtischen (aber vielleicht den Beginn eines besseren Verständnisses unserer Welt). Kommentar zu Stefan Höhne und Boris Michel. In: sub\urban. zeitschrift für kritische stadtforschung 9/1-2, 151-157.

SRF (2020): Indien wird mit voller Wucht von zweiter Welle getroffen. 14.4.2021. https:// www.srf.ch/news/international/coronavirus-indien-wird-mit-voller-wucht-von-zweiterwelle-getroffen (letzter Zugriff am 10.5.2021).

Strack, Christoph (2021): Corona-Massenausbruch: „Zweierlei Maß“ bei Erntehelfern. In: Deutsche Welle, 4.5.2021. https://www.dw.com/de/corona-massenausbruch-zweierleima\%C3\%9F-bei-erntehelfern/a-57424712 (letzter Zugriff am 6.5.2021).

Strüver, Anke (2021): The end of care-less capitalism (as we knew it)? Kommentar zu Stefan Höhne und Boris Michel. In: sub\urban. zeitschrift für kritische stadtforschung 9/1-2, 165-170.

Verma, Anshul (2020): Coronavirus: Huge crowds as India lockdown sparks mass migration. In: BBC News, 30.3.2020. https://www.bbc.com/news/av/world-asia-india-52093574 (letzter Zugriff am 10.5.2021).

Wachtler, Benjamin / Michalski, Niels / Nowossadeck, Enno / Diercke, Michaela / Wahrendorf, Morten / Santos-Hövener, Claudia / Lampert, Thomas / Hoebel, Jens (2020a): Socioeconomic inequalities and Covid-19 - A review of the current international literature. In: Journal of Health Monitoring 5/S7, 3-17.

Wachtler, Benjamin / Michalski, Niels / Nowossadeck, Enno / Diercke, Michaela / Wahrendorf, Morten / Santos-Hövener, Claudia / Lampert, Thomas / Hoebel, Jens (2020b): Sozioökonomische Ungleichheit im Infektionsrisiko mit SARS-CoV-2 erste Ergebnisse einer Analyse der Meldedaten für Deutschland. In: Journal of Health Monitoring 5/S7, 18-29.

Wahrendorf, Morten / Rupprecht, Christoph J. / Dortmann, Olga / Scheider, Maria / Dragano, Nico (2021): Erhöhtes Risiko eines Covid-19-bedingten Krankenhausaufenthaltes für Arbeitslose: Eine Analyse von Krankenkassendaten von 1,28 Mio. Versicherten in Deutschland. In: Bundesgesundheitsblatt, Gesundheitsforschung, Gesundheitsschutz $64 / 3,314-321$.

WHO (2020): Senegal reports first COVID-19 case. World Health Organization 2.3.2020. https://www.afro.who.int/news/senegal-reports-first-covid-19-case (letzter Zugriff am 6.5.2021).

Zinn, Sabine / Kreyenfeld, Michaela / Bayer, Michael (2020): Kinderbetreuung in CoronaZeiten: Mütter tragen die Hauptlast, aber Väter holen auf. In: DIW aktuell 51. 